\title{
A tubby-like protein CsTLP8 acts in the ABA signaling pathway and negatively regulates osmotic stresses tolerance during seed germination
}

Shuangtao $\mathrm{Li}^{1,2+}{ }^{4}$, Zhirong Wang ${ }^{1 \dagger}$, Fei Wang ${ }^{1}$, Hongmei Lv ${ }^{1}$, Meng Cao ${ }^{1}$, Na Zhang ${ }^{1}$, Fengju Li ${ }^{3}$, Hao Wang ${ }^{3}$, Xingsheng $\mathrm{Li}^{4}$, Xiaowei Yuan ${ }^{4}$, Bing Zhao ${ }^{1 *}$ and Yang-Dong Guo ${ }^{1^{*}}$

\begin{abstract}
Background: TLPs (Tubby-like proteins) are widespread in eukaryotes and highly conserved in plants and animals. TLP is involved in many biological processes, such as growth, development, biotic and abiotic stress responses, while the underlying molecular mechanism remains largely unknown. In this paper we characterized the biological function of cucumber (Cucumis sativus L.) Tubby-like protein 8 (CsTLP8) in Arabidopsis.

Results: In cucumber, the expression of the tubby-like protein CSTLP8 was induced by $\mathrm{NaCl}$ treatment, but reduced by PEG (Polyethylene Glycol) and ABA (Abscisic Acid) treatment. Subcellular localization and transcriptional activation activity analysis revealed that CSTLP8 possessed two characteristics of classical transcription factors: nuclear localization and trans-activation activity. Yeast two-hybrid assay revealed interactions of CsTLP8 with CsSKP1a and CsSKP1C, suggesting that CSTLP8 might function as a subunit of E3 ubiquitin ligase. The growth activity of yeast with ectopically expressed CSTLP8 was lower than the control under $\mathrm{NaCl}$ and mannitol treatments. Under osmotic and salt stresses, overexpression of CSTLP8 inhibited seed germination and the growth of Arabidopsis seedlings, increased the content of MDA (Malondialdehyde), and decreased the activities of SOD (Superoxide Dismutase), POD (Peroxidase) and CAT (Catalase) in Arabidopsis seedlings. Overexpression of CSTLP8 also increased the sensitivity to ABA during seed germination and ABA-mediated stomatal closure.
\end{abstract}

Conclusion: Under osmotic stress, CsTLP8 might inhibit seed germination and seedling growth by affecting antioxidant enzymes activities. CsTLP8 acts as a negative regulator in osmotic stress and its effects may be related to ABA.

Keywords: Cucumis sativus L, E3 ubiquitin ligase, Salt stress, Osmotic stress, Transcription factor, Tubby-like protein

\footnotetext{
*Correspondence: zhaobing@cau.edu.cn; yaguo@cau.edu.cn

†'Shuangtao Li and Zhirong Wang contributedequally to this work.

${ }^{1}$ Beijing Key Laboratory of Growth and Developmental Regulation

for Protected Vegetable Crops, College of Horticulture, China Agricultural

University, Beijing 100193, China

Full list of author information is available at the end of the article
}

(C) The Author(s) 2021. Open Access This article is licensed under a Creative Commons Attribution 4.0 International License, which permits use, sharing, adaptation, distribution and reproduction in any medium or format, as long as you give appropriate credit to the original author(s) and the source, provide a link to the Creative Commons licence, and indicate if changes were made. The images or other third party material in this article are included in the article's Creative Commons licence, unless indicated otherwise in a credit line to the material. If material is not included in the article's Creative Commons licence and your intended use is not permitted by statutory regulation or exceeds the permitted use, you will need to obtain permission directly from the copyright holder. To view a copy of this licence, visit http://creativecommons.org/licenses/by/4.0/. The Creative Commons Public Domain Dedication waiver (http://creativeco mmons.org/publicdomain/zero/1.0/) applies to the data made available in this article, unless otherwise stated in a credit line to the data. 


\section{Background}

Tubby was first identified in the tubby strain of obese mice [1, 2]. TLPs are widespread in eukaryotes [3]. TLPs contain an about 270-amino acid tubby domain at the $\mathrm{COOH}$-terminal, with a structure containing 12 anti-parallel $\beta$ barrels and an intermediate hydrophobic $\alpha$ helix [4]. The $\mathrm{NH}_{2}$-terminal sequences of TLPs are quite divergent in animals, but conserved in plants, with most plant TLPs containing a conserved F-box domain in the $\mathrm{NH}_{2}$-terminal [3]. SKP1 (S-phase kinaseassociated protein 1), CUL (Cullin), RBX1 (RING-box protein 1), and F-box protein can form the SCF complex $[5,6]$, an important component of E3 ubiquitin ligase. In Arabidopsis and wheat, AtTLPs and TaTULPs have been confirmed to interact with specific SKP1-like proteins [7-10], these findings indicate that plant TLPs might function as subunits of SCF complexes. In mammals, Tubby-like proteins constitute a unique family of transcriptional regulators [4], as the $\mathrm{COOH}$-terminal region of Tubby binds double-stranded DNA, and the $\mathrm{NH}_{2}$-terminal regions of Tubby and TULP1 activate transcription. In plants, AtTLPs and CaTLP1 lack autoactivation activity $[7,11]$, but CaTLP1 binds to doublestranded DNA [11], suggesting that CaTLP1 may be a transcription factor.

Previous studies have assessed the efficacy of TLPs in response to biotic and abiotic stress in plants. In rice, all OsTLPs might involve in plant-pathogen interaction [12], besides, OsTLP2 can bind to the promoter of OsWRKY13, which encodes an activator important in rice resistance to bacterial infection [13], suggesting that OsTLP2 might function as a transcription factor to regulate biotic stress tolerance. In coffee and sugarcane TLPS were provided that play a role in response to biotic stress $[14,15]$.

In medicago, barley, maize, cassava, apple and chickpea, TLPs were reported to play a role in response to abiotic stress treatments or hormone treatment [11, 16-20]. Ectopically expressed MdTLP7 increased growth activity of $E$. coli under $\mathrm{NaCl}, \mathrm{KCl}$, chilling, and heat treatment [21], and overexpression of full length or truncated MdTLP7 (containing only the tubby domain) enhanced the stress tolerance of Arabidopsis to abiotic stresses [22], suggesting that $M d T L P 7$ functions in response to abiotic stress and the tubby domain of MdTLP7 plays a key role in this response. The expression of CaTLP1 was induced by dehydration, high salinity, and ABA [11]. Overexpression of CaTLP1 increased the tolerance of tobacco and Arabidopsis to abiotic stress [11, 23]. Further study implied that CaTLP1 regulates the expression of ABA-mediated genes and stomatal closure by interacting with protein kinase [23]. AtTLP3 was released from the plasma membrane under mannitol, $\mathrm{NaCl}$, or $\mathrm{H}_{2} \mathrm{O}_{2}$ treatment, suggesting that AtTLP3 functions in response to osmotic stress $[7,24]$.

In addition to response to biotic and abiotic stress, TLP also plays a role in plant growth and development. AtTLP9 functions during seed germination and participates in the ABA signaling pathway [9], and AtTLP3 redundantly functions with AtTLP9 in ABAand osmotic stress-mediated seed germination [7]. The overexpression of CaTLP1 improved shoot and root architecture, suggesting a key role for CaTLP1 in plant development [11]. AtTLP2 regulates the biosynthesis of homogalacturonan [25], the major polysaccharide constituent of Arabidopsis seed coat mucilage [26], possibly through positive activation of UDP-glucose 4-epimerase 1 [25]. AtTLP11 interacts with AtNHL6, AtTLP11 and AtNHL6 exhibit antagonistic gene expression during seed germination [27]. AtNHL6 functions in abiotic stress-induced ABA signal transduction and biosynthesis, especially during early seedling development and seed germination $[27,28]$, and AtTLP11 may function by regulating AtNHL6. ScTLP12 was identified as a putative gene responsible for leaf rolling in rye [29]. Zhang et al. explored the expression of SlTLPs in tomato fruit founded that SITLP1, SITLP2, SITLP4 and SITLP5 are ripening-related, and SITLP1 and SITLP2 may play a role in ethylene-dependent fruit ripening [30]. Our previous works imply that SITLFP8 regulated stomatal density through nuclear endoreduplication and affected water-deficient resistance and water-use efficiency [31].

Seed germination is a key process in the life cycle of higher plants, and play a function role in plant adapt to various environmental conditions, meanwhile, seed germination is stringently regulated by external and internal cues such as light, temperature, water, oxygen, plant hormone ABA, and GA [32-34]. Among these, the phytohormone abscisic acid (ABA) functions as a crucial signal to inhibit seed germination [35]. Drought and salt stresses are common limiting factors of seed germination. Drought and salt stress affects the seed germination process by decreasing water imbibition, including ROS (Reactive Oxygen Species) accumulation, altering enzymatic activities, and causing hormonal imbalances [36-38].

In this study, we found that CsTLP8 transcriptionally responded to PEG, $\mathrm{NaCl}$, and ABA treatments. Overexpression of CsTLP8 inhibited the growth of yeast cells under $\mathrm{NaCl}$ and mannitol treatments. Overexpression of CSTLP8 inhibited seed germination and the growth of Arabidopsis seedlings under osmotic and salt stresses, and increased ABA sensitivity in Arabidopsis. Under $\mathrm{ABA}$, mannitol and $\mathrm{NaCl}$ treatment, overexpression of CsTLP8 increased the content of 
MDA, and decreased the activities of SOD, POD and CAT in Arabidopsis seedlings. These results indicate that CsTLP8 acts as a negative regulator in salt stress and osmotic stress response by affecting antioxidant enzymes activities. Subcellular localization and transactivation assays suggested CsTLP8 works as a transcription factor, and yeast two-hybrid assays revealed interaction of CsTLP8 with CsSKP1a and CsSKP1c, implying that CsTLP8 may also function as an E3 ubiquitin ligase.

\section{Results}

\section{CSTLP8 expression responds to abiotic stress}

To investigate the function of CsTLP8 under abiotic stress in cucumber, we analyzed the expressions of CsTLP8 under $\mathrm{NaCl}$, PEG and ABA treatments. The expression level of CSTLP8 was up-regulated about sixfold at $9 \mathrm{~h}$ after $\mathrm{NaCl}$ treatment (Fig. 1a), and reduced under both PEG treatment (Fig. 1b) and ABA treatment (Fig. 1c). Interestingly, the expression pattern of CsTLP8 under ABA or PEG treatment was similar. We next explored the expression pattern of CsTLP8 in seven organs (root, stem, leaf, staminate flower, pistillate flower and fruit).
Results showed that CsTLP8 was expressed in all the organs tested, the expression level of CsTLP8 was highest in staminate flower and lowest in fruit, and there was no significant difference in the expression level of CsTLP8 in vegetative organs, but significant differences in reproductive organs (Fig. 1d).

\section{Structural characteristics of CsTLP8}

To better understand the transcriptional regulation of CsTLP8, we extracted 2000 bp upstream sequences of CsTLP8 and searched the PlantCARE database to identify the cis-elements. The CsTLP8 promoter contains various cis-acting elements, including stress-responsive elements: TC-rich repeats (defense and stress response), MBS (drought response), GT-1 motif (salt response), LTR (temperature response), and ARE (anaerobic induction); hormone response elements: TCA-element (salicylic acid response), ABRE (Abscisic acid response), TGACG/ CGTCA-motif (Methyl Jasmonate response); and light response elements: G-Box, MRE, AE-box, TCT-motif, and LAMP-element (Fig. 2c).

To better understand the function of CsTLP8, we analyzed its structural features. Conserved domain
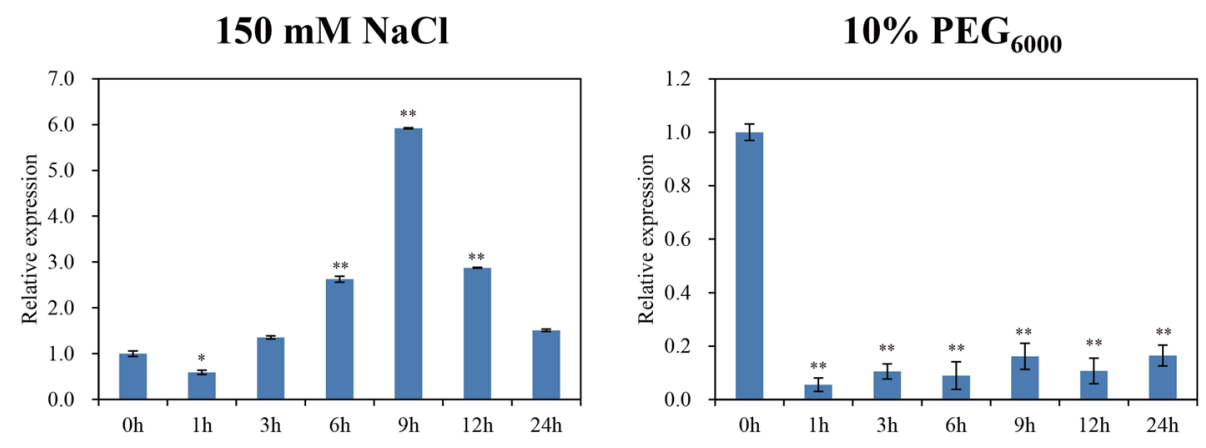

$100 \mu \mathrm{M}$ АBA

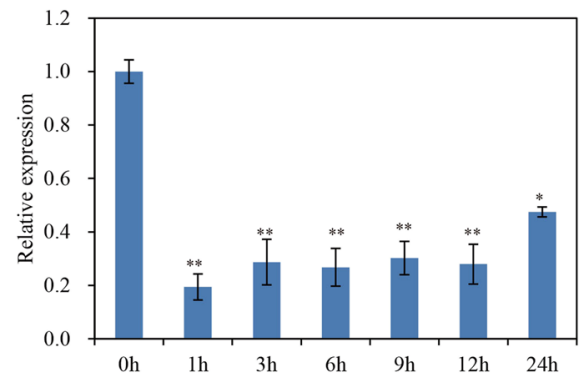

Organs

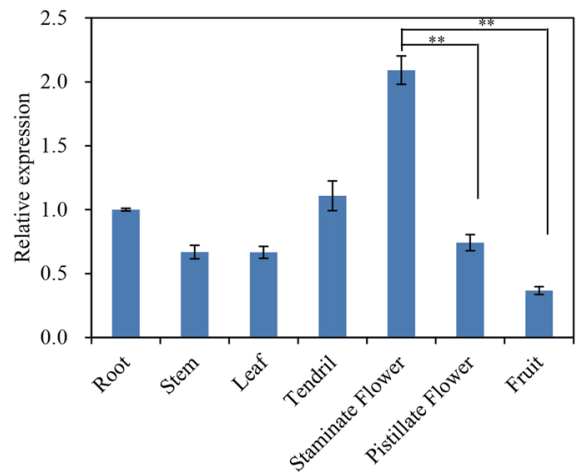

Fig. 1 The expression pattern of CSTLP8. (a) The expression pattern of CSTLP8 in the leaves under NaCl (150 mM) treatment. (b) The expression pattern of CSTLP8 in the leaves under PEG 6000 (10\%) treatment. (c) The expression pattern of CSTLP8 in the leaves under ABA (100 $\mu M)$ treatments. (d) The expression pattern of CSTLP8 in various organs (root, stem, leaf, staminate flower, pistillate flower, fruit and tendril). Values are means \pm SD $(\mathrm{n}=3)$, ${ }^{*} P<0.05$ or ${ }^{* *} P<0.01$, Duncan's test for multiple tests 
analysis showed that CsTLP8 harbor an F-box domain in the $\mathrm{NH}_{2}$-terminal region and a tubby domain in the $\mathrm{COOH}$-terminal region (Fig. 2a). The 3D model of CsTLP8 revealed a highly conserved tubby domain, consisting of a closed 12-stranded $\beta$ barrel and a central $\alpha$ helix (Fig. S1), the typical structure of the tubby domain. Phylogenetic analysis showed that CsTLP8 belongs to group A, and clustered in a clade with
MeTLP3 and MeTLP13 (Fig. 2a). The presence of the F-box domain in the $\mathrm{NH}_{2}$-terminal region suggested that CsTLP8 may function as a subunit of the SCF complex. Multiple sequence alignment of CsTLP8 and AtTLPs revealed that CsTLP8 contains a conserved $\mathrm{PIP}_{2}$ (Phosphatidylinositol 4, 5-bisphosphate) binding site ( Lys $_{188} / \mathrm{Arg}_{190}$ ) (Fig. 2b), which has previously shown to play a core role in the plasma membrane

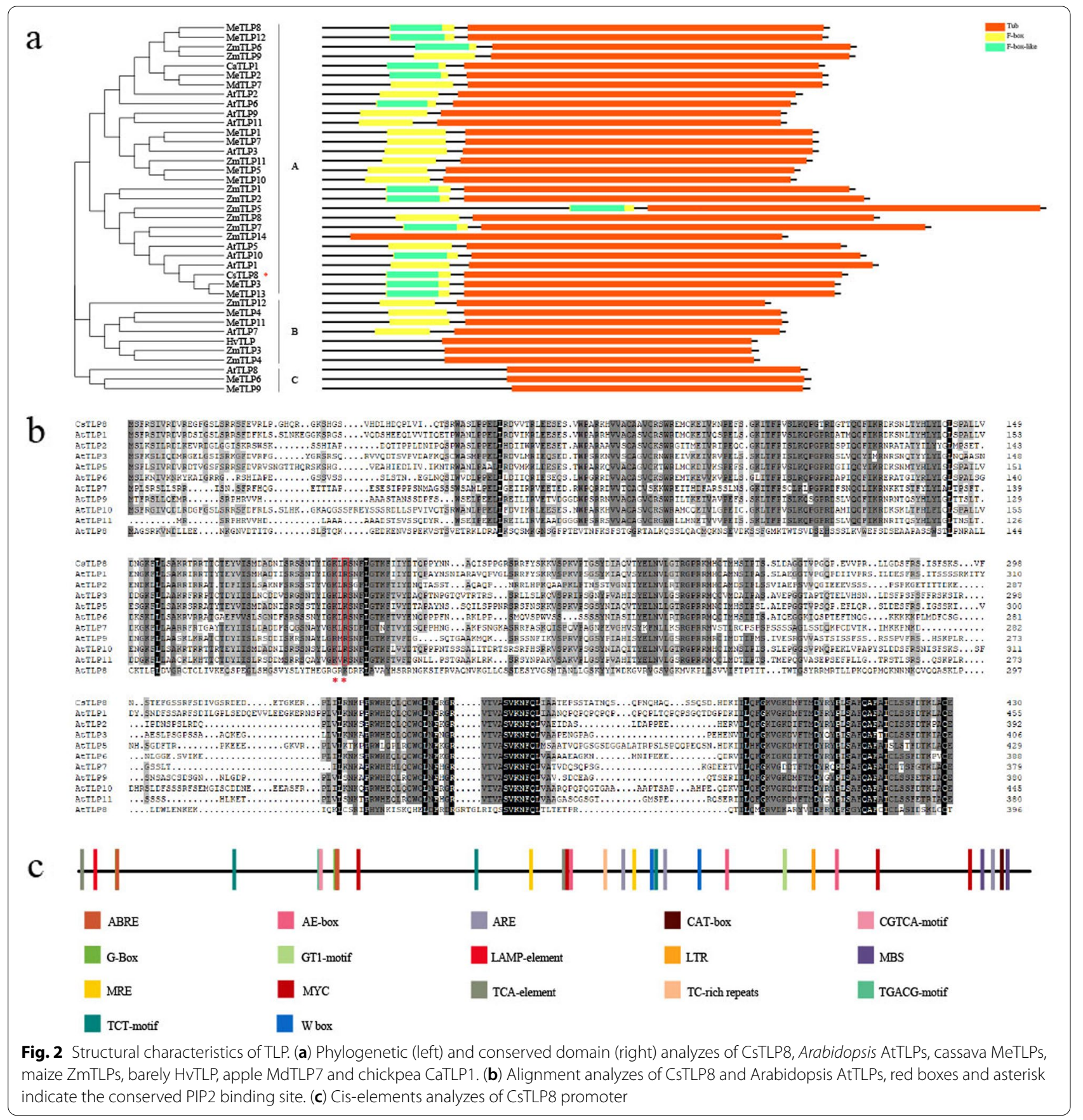


localization of Tubby [39] and AtTLP3 [24], indicating that CsTLP8 might locate in the plasma membrane.

\section{The subcellular localization and transactivation activity of CsTLP8}

To further investigate the function of CsTLP8, we examined the subcellular localization of CsTLP8 in tobacco leaves. The fluorescence of CsTLP8-GFP fusion protein was observed in plasma membrane and nucleus, indicating that CsTLP8 localized in the plasma membrane and nucleus (Fig. 3), which is consistent with our conjecture.

To detect whether CsTLP8 had a transcription activation function, we analyzed the potential transcriptional activation activity of CsTLP8 by yeast one-hybrid assay. After incubated for 3 days, yeast cells transformed with pGBKT7-CsTLP8 or pGBKT7-CsATAF1 (positive control) survived well in $\mathrm{SD} /-\mathrm{Trp} /$-His/X- $\alpha$-gal medium and turned blue, indicating activation of the reporter genes (Fig. 4). This result showed that CsTLP8 has auto-activation activity, and combined with the nuclear localization of CsTLP8, it is likely that CsTLP8 can function as a transcription factor in cucumber.

\section{The growth performance of yeast strains carrying CsTLP8} decreases under osmotic and salt stresses

To assess the role of CsTLP8 under osmotic and salt stresses, CsTLP8-overexpressing yeast cells were generated, and the growth performance of these transgenic yeast cells was examined under osmotic and salt stresses by spot assays. Under normal conditions, there was no significant difference in growth performance for yeast cells transformed with pYES2-CsTLP8 or empty vector pYES2 (control) (Fig. 5a). However, the growth of CsTLP8overexpressing yeast cells was weaker than the control on medium supplemented with $250 \mathrm{mM} \mathrm{NaCl}$ or $300 \mathrm{mM}$ mannitol (Fig. 5b-c), suggesting that overexpressing of CsTLP8 significantly reduced the abiotic stress tolerance of yeast cells.

\section{Overexpression of CSTLP8 decreases the tolerance to osmotic and salt stresses during seed germination in Arabidopsis}

To further investigate the biological function of CsTLP8, we ectopically expressed the coding sequences of CSTLP8 in wild type Arabidopsis (Col-0). Two transgenic lines, OE7 and OE11, were selected for further study (Fig. S2). As shown above, the expression of CsTLP8 responded to PEG and $\mathrm{NaCl}$ treatments, and ectopic expression of CsTLP8 reduced growth performance of yeast cells under osmotic and salt stresses. Therefore, we measured the seed germination and seedling root length of wild type plants and transgenic lines on MS medium supplemented with different concentrations of mannitol and $\mathrm{NaCl}$. Under normal conditions, the seedling growth and seed germination rate of transgenic lines were similar to those of wild type (Fig. 6). Under mannitol and $\mathrm{NaCl}$ treatment, the inhibition of seed germination and seedling growth of transgenic lines were more significant than those of wild lines. On medium containing $75 \mathrm{mM}$ mannitol, the germination rates of wild type and two transgenic lines were 96\%, 78\%, and 82\%, respectively (Fig. S3

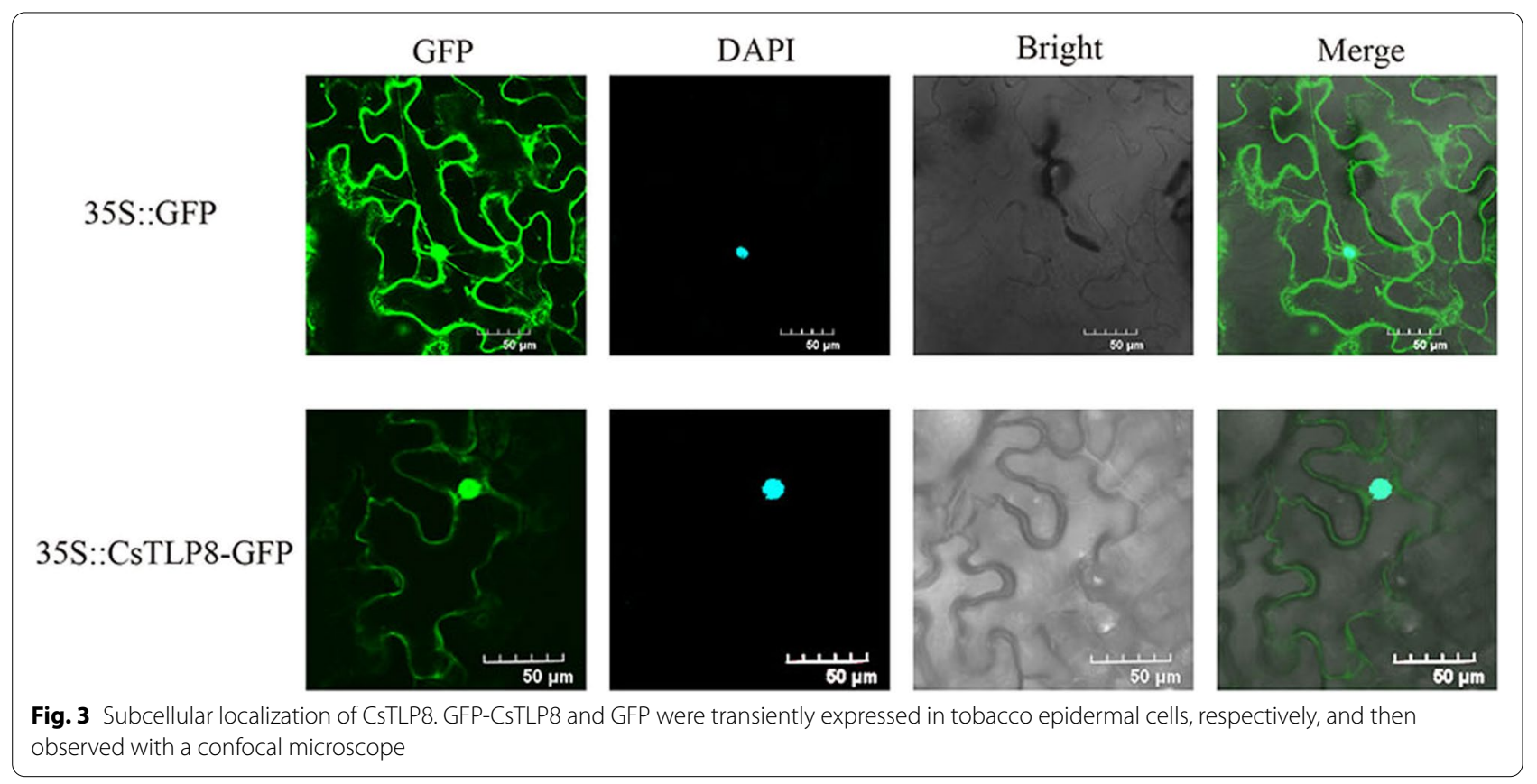




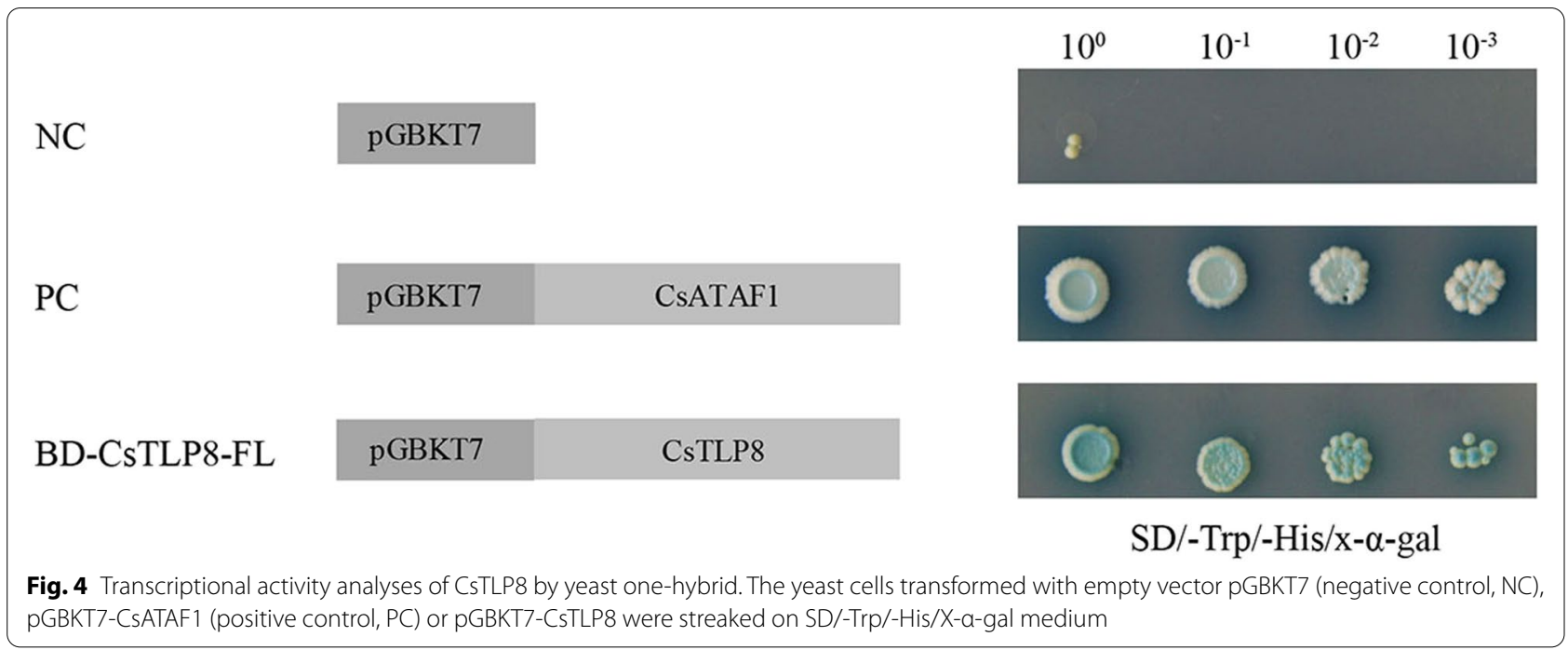

\& Fig. 6b), and the length of root were $16.0 \mathrm{~mm}, 11.3 \mathrm{~mm}$ and $10.5 \mathrm{~mm}$, respectively (Fig. S3 \& Fig. 6c-d). With the increase of mannitol concentration, the inhibition of seed germination increased. On the medium containing $100 \mathrm{mM}$ mannitol, the germination rates of wild type and two transgenic lines were $74 \%, 49 \%$ and $50 \%$, respectively, (Fig. 6a-b), and the root lengths were $10.7 \mathrm{~mm}$, $5.9 \mathrm{~mm}$, and $5.3 \mathrm{~mm}$, respectively (Fig. 6c-d). At $75 \mathrm{mM}$ $\mathrm{NaCl}$, the germination rates of wild type and transgenic lines were $93 \%, 70.4 \%$ and $70.2 \%$, respectively, (Fig. S3 \& Fig. 6b) and root lengths of wild type and transgenic lines were $15.5 \mathrm{~mm}, 11.0 \mathrm{~mm}$, and $12.2 \mathrm{~mm}$, respectively (Fig. S3 \& Fig. 6c-d). At $100 \mathrm{mM} \mathrm{NaCl}, 34 \%-38 \%$ seeds of transgenic lines germinated, while $78 \%$ seeds of wild type germinated (Fig. 6a-b) and the root lengths of wild type and transgenic lines were $10.3 \mathrm{~mm}, 5.3 \mathrm{~mm}$ and $4.6 \mathrm{~mm}$, respectively (Fig. 6c-d). All these results indicated that CsTLP8 functions during seed germination and seedling growth in Arabidopsis.

\section{Overexpression of CSTLP8 increases ABA sensitivity in Arabidopsis}

As the expression of CsTLP8 responded to ABA treatments, we next measured the response to $\mathrm{ABA}$ in transgenic lines. We employed seed germination rate, seedling root length and stomata aperture as indicators of ABA sensitivity. The seeds of wild type and transgenic lines germinated on MS medium supplemented with different concentrations of ABA. Under normal conditions, the seed germination rate and seedling growth of transgenic lines were similar to those of wild type. With the increase of ABA concentration, the seed germination rate decreased and the inhibition on seedling growth increased. There was a significant difference in seedling growth between transgenic lines and wild type, although there was no significant difference in seed germination rate between CsTLP8 overexpression plants and wild type (Fig. 6a-b and Fig. S3). At $0.1 \mu \mathrm{M} \mathrm{ABA}$, the root length of wild type was 1.41.5 times that of transgenic lines (Fig. S3 \& Fig. 6c-d). The seedling growth difference was more significant at $0.25 \mu \mathrm{M} \mathrm{ABA}$, and the root length of wild type was 1.8 2.3 times that of transgenic lines (Fig. 6c-d). To further examine the function of CsTLP8 to the ABA response, we measured stomatal apertures in transgenic plants, and wild-type plants in response to ABA. There was no difference in stomatal aperture of all the lines in the absence of $A B A$, but ABA treatment led to an increased stomatal closure in the transgenic lines, in the presence of $5 \mu \mathrm{M} \mathrm{ABA}$, the stomatal aperture of wild type was 1.4-1.5 times that of transgenic lines; in the presence of $10 \mu \mathrm{M} \mathrm{ABA}$, the stomatal aperture of wild type was 1.5-1.6 times that of transgenic lines (Fig. 7). These data suggesting that CsTLP8 positively regulates ABAdependent stomatal movement. These results showed that overexpression of CsTLP8 increased the sensitivity to ABA in Arabidopsis.

\section{Overexpression of CSTLP8 reduce antioxidant enzymes activities in Arabidopsis seedling under osmotic and salt stresses}

To evaluate physiological changes, the contents of MDA and activities of SOD, POD, CAT were measured following ABA, mannitol and salt treatment. Under normal conditions, there was no significant difference in MDA content between wild type and transgenic lines; under ABA, mannitol and salt treatment, the content of MDA in wild type and transgenic lines increased, and 
a

b

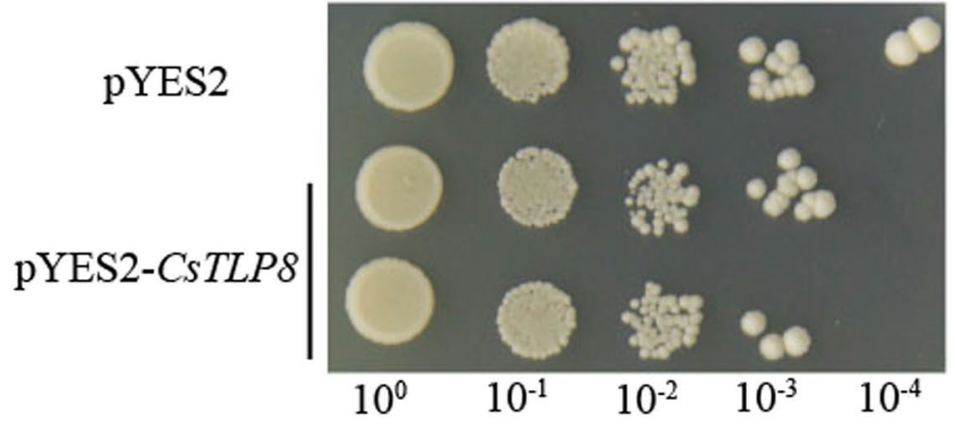

Fig. 5 Transformed yeast strains had reduced growth activity under salt and drought stresses. The growth activity of yeast cells was tested by yeast spot assay when grown under control (a), $250 \mathrm{mM} \mathrm{NaCl}$ (b) and $300 \mathrm{mM}$ mannitol (c) conditions on solid media

the content of MDA in wild type was significantly higher than that of transgenic lines (Fig. 8a). Under normal conditions, there was no significant difference in SOD, POD and CAT activities between wild type and transgenic lines; under ABA, mannitol and salt treatment, the activities of SOD, POD and CAT in all lines increased, and the activities of SOD, POD and CAT in transgenic lines were significantly higher than those of wild type (Fig. 8b-8c).

\section{CsTLP8 interacts with CsSKP1a and CsSKP1c}

We performed yeast two-hybrid assay to ask if CsTLP8 could interact with CsSKP1s as an F-box protein. The results suggest that CsTLP8 can interact with CsSKP1a and CsSKP1c (Fig. 9), indicating that CsTLP8 may function as an F-box protein, a subunit of SCF complex and participate in the degradation of the target protein.

\section{Discussion}

CsTLP8 was previously identified as a drought-related gene given its higher expression in drought-sensitive cultivar [40]. In our study, we found that expression of CSTLP8 responded to salt and osmotic stresses (Fig. 1), overexpression of CSTLP8 significantly reduced resistance of yeast cells to salt and osmotic stresses (Fig. 5), 
a

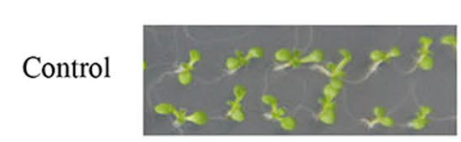

$\mathrm{ABA}$

$0.25 \mu \mathrm{M}$

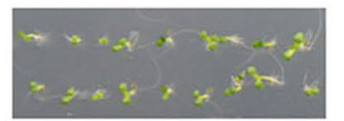

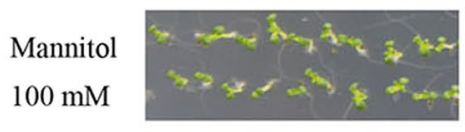

$\mathrm{NaCl}$

$100 \mathrm{mM}$

WT
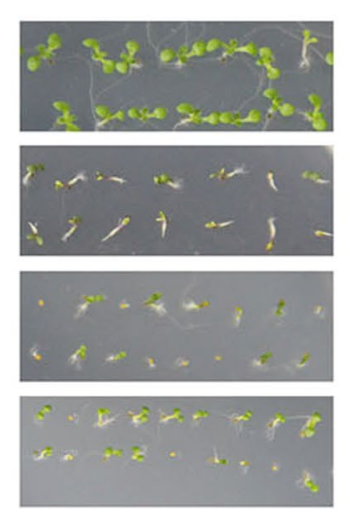

OE11
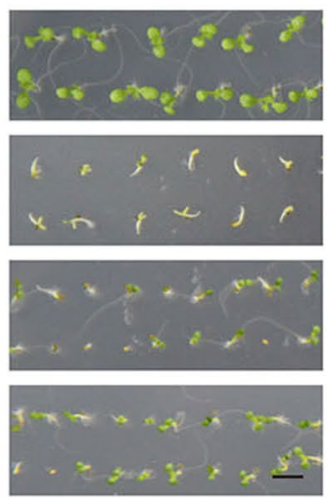

b

Germination rate

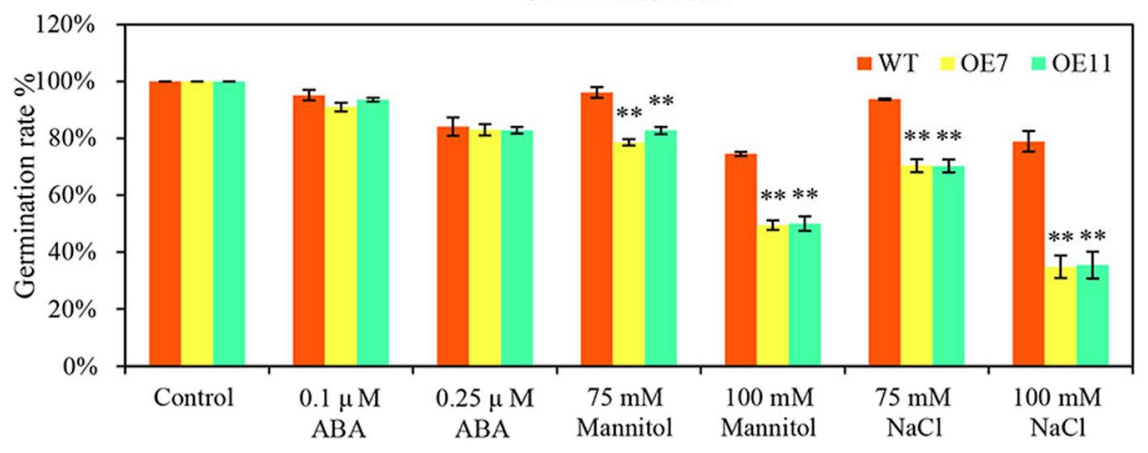

c

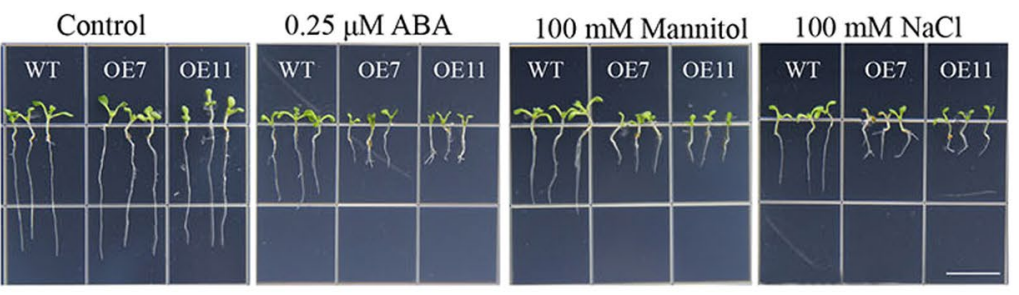

d

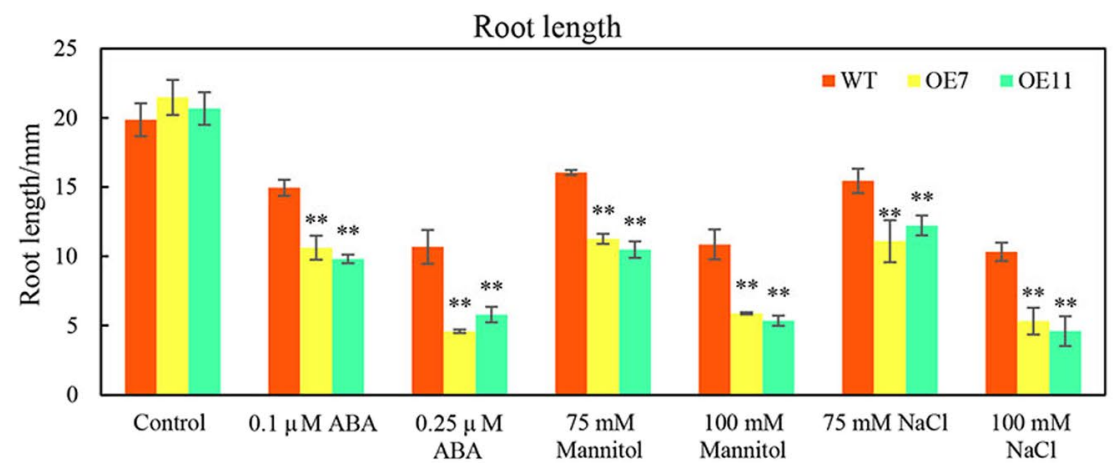

Fig. 6 Overexpression of CSTLP8 increased the sensitivity to ABA and decreased the tolerance to osmotic and salt stresses during seed germination in Arabidopsis. (a) Seeds of Col-0, OE7 and OE11 were surface sterilized, stratified for $3 \mathrm{~d}$, and germinated on 1/2 MS media supplemented without or with $\mathrm{ABA}$, mannitol or $\mathrm{NaCl}$. Bar $=1 \mathrm{~cm}$. (b) Quantification of corresponding germination rates. (c) Seeds of Col-0, OE7 and OE11 were sown for 4 days on half-strength MS medium and then transferred to half-strength MS medium with different concentrations of $\mathrm{ABA}$, $\mathrm{NaCl}$, or mannitol for 7 days. Bar $=1 \mathrm{~cm}$. (d) Quantification of corresponding seedling root length. Values are means $\pm S D(n=3)$, ${ }^{*} P<0.05$ or ${ }^{* *} P<0.01$, Duncan's test for multiple tests 


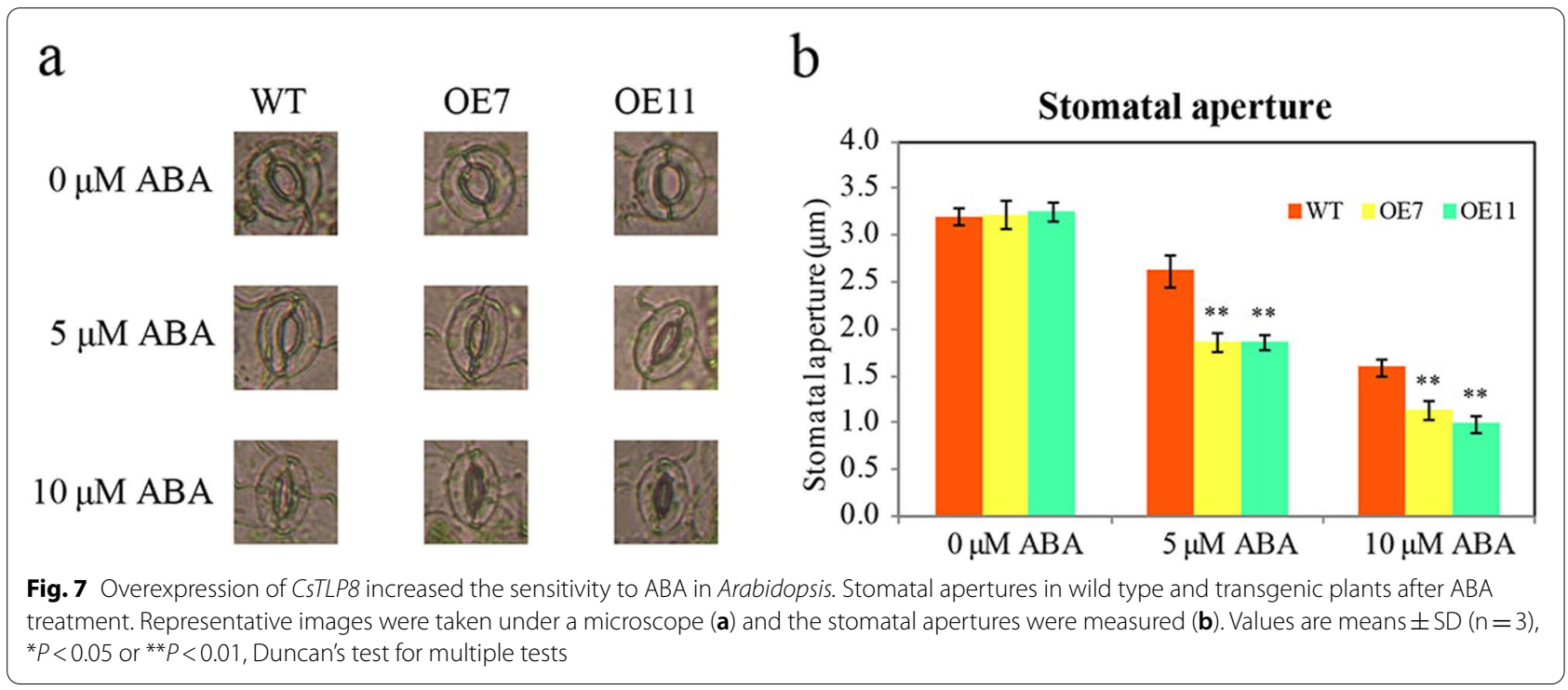

and seed germination and seedling growth were inhibited in CSTLP8 overexpression plants under salt and osmotic stresses (Fig. 6 and Fig. S3). These findings implied that CsTLP8 participates in the abiotic stress response during seed germination and seedling establishment. The process of germination occurs in three main phases regulated by hormones, reactive nitrogen species, and ROS [41]. ROS function as signaling molecules to regulate plant growth and development, but excess ROS could damage the structures of DNA, protein, lipid, and other macromolecules in the seeds. Thus, ROS scavenging is pivotal for seed germination under stress

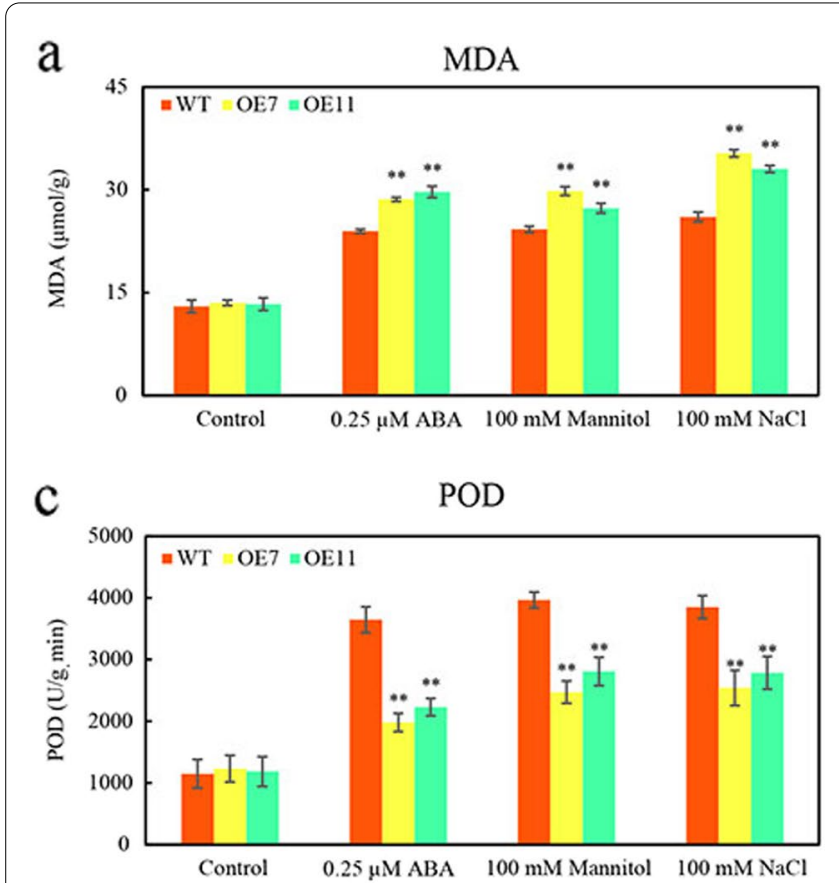

b

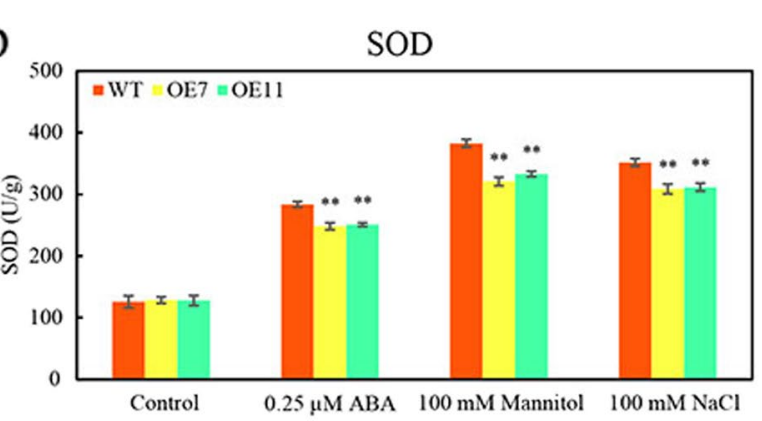

d

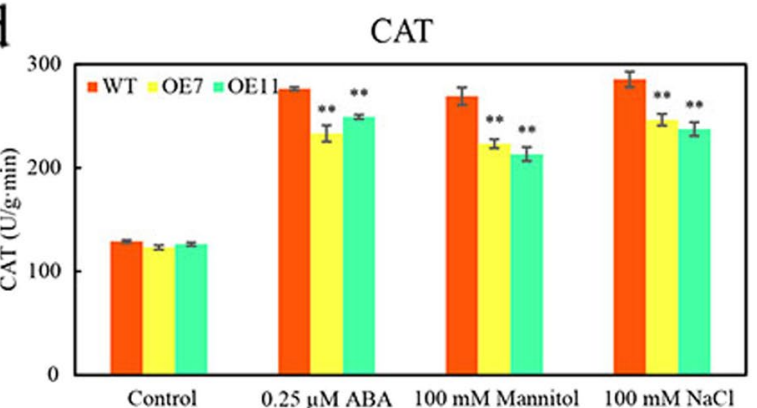

Fig. 8 Overexpression of CSTLP8 reduce antioxidant enzymes activities in Arabidopsis seedling under osmotic and salt stresses. (a) The content of MDA (Malondialdehyde) in wild type and transgenic lines under $0.25 \mu \mathrm{M} \mathrm{ABA}, 100 \mathrm{mM}$ mannitol and $100 \mathrm{mM} \mathrm{NaCl}$ treatment. (b) The activity of SOD (Superoxide Dismutase) in wild type and transgenic lines under $0.25 \mu \mathrm{M} \mathrm{ABA}, 100 \mathrm{mM}$ mannitol and $100 \mathrm{mM} \mathrm{NaCl}$ treatment. (c) The activity of POD (Peroxidase) in wild type and transgenic lines under $0.25 \mu \mathrm{M} \mathrm{ABA}, 100 \mathrm{mM}$ mannitol and $100 \mathrm{mM} \mathrm{NaCl}$ treatment. (d) The activity of CAT (Catalase) in wild type and transgenic lines under $0.25 \mu \mathrm{M} \mathrm{ABA}, 100 \mathrm{mM}$ mannitol and $100 \mathrm{mM} \mathrm{NaCl}$ treatment. Values are means \pm SD $(n=3)$, ${ }^{*} P<0.05$ or ${ }^{* *} P<0.01$, Duncan's test for multiple tests 


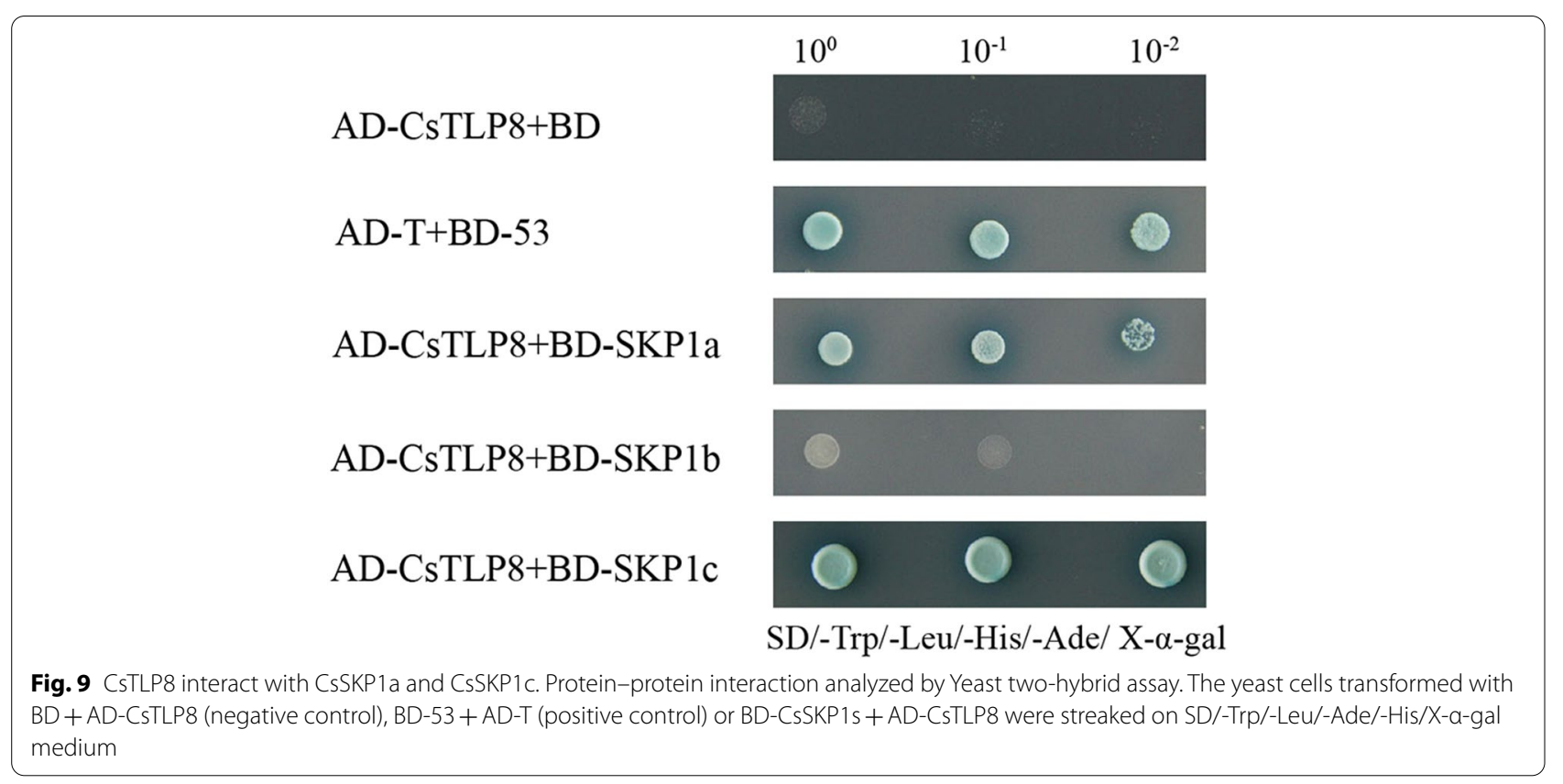

conditions $[42,43]$. ROS can induce lipid peroxidation, which results in the production of MDA. In our study the content of MDA was used as a marker of oxidative stress, we found that overexpression of CsTLP8 increased the level of oxidative stress in Arabidopsis seedling under osmotic and salt stresses. On the other hand, overexpression of CSTLP8 reduced antioxidant enzymes activities in Arabidopsis seedling under osmotic and salt stresses. These results indicated that CsTLP8 might regulate seed germination under abiotic stress by affecting antioxidant enzymes activities. As a crucial phytohormone, ABA plays a key role in many aspects of plants, including seed dormancy and germination, root growth, seedling development, and the adaptive response to environmental stresses. Overexpression of CsTLP8 increased the sensitivity to ABA in Arabidopsis, such as shorter seedling root length and smaller stomatal aperture (Fig. 6, 7 and Fig. S3).

Cis-acting regulatory elements play key roles in the control of gene expression, and the CsTLP8 promoter region contains various stress- and hormone-response elements (Fig. 2c). The observed different expression patterns under salt and PEG treatments (Fig. 1a-1b) suggest that the expression of CSTLP8 can be regulated by different transcription factors via binding to different cis-acting elements under different stress signals. In Arabidopsis, AtTLP3 and AtTLP9 redundantly function during seed germination and early seedling development [7]. The phenotypes of AtTLP3 knockout mutants and AtTLP3 and AtTLP9 double mutants under ABA and osmotic stress during seed germination are opposite to that of plants overexpressing CsTLP8, implying that CsTLP8 plays a similar role during seed germination to AtTLP3 and AtTLP9. Phylogenetic tree analysis clustered CsTLP8 in a clade with MeTLP13 and MeTLP3, and interestingly, the expression patterns of CsTLP8 under $\mathrm{NaCl}$ treatment (Fig. 1a) is similar to that of MeTLP3 as described by Dong et al. [19].

Structural features analysis and the 3D model revealed that CsTLP8 is a typical plant TLP (Fig. 2a), Multiple sequence alignment showed that CsTLP8 possesses a conserved PIP2 binding site (Fig. 2b), indicating that CsTLP8 may be located on the plasma membrane, which was confirmed by the results shown in Fig. 3. Tubby-PIP2 binding is reversible, as Tubby can interact with $\mathrm{G \alpha}_{\mathrm{q}}$ a subset of G-proteins, resulting in the release of Tubby from the plasma membrane and enabling its translocation to the nucleus [39]. In Arabidopsis, AtTLP2 can interact with NF-YC3 (Nuclear Factor Y subunit C3) and be translocated into the nucleus [25]. AtTLP3 was released from plasma membrane under mannitol, $\mathrm{NaCl}$, and $\mathrm{H}_{2} \mathrm{O}_{2}$ treatment [7], and the accumulation of CaTLP1 in nucleus was induced by dehydration stress [11].

In our study, we confirmed that CsTLP8 has transactivation activity (Fig. 4) and combined with the nuclear localization of CsTLP8 (Fig. 3), we speculate that CsTLP8 may act as a transcription factor in cucumber. Most plant TLPs contains an F-box domain, like CsTLP8. Previous studies showed that most AtTLPs (AtTLP1, 3, 6, 9, 10 and 11) and TaTLPs (TaTULP1, TaTULP3 and TaTULP4) can interact with specific ASKs (Arabidopsis Skp1-like proteins)/ TaSKPs $[8,10]$. Consistent with those findings, 
our research has demonstrated that CsTLP8 can interact with CsSKP1a and CsSKP1c (Fig. 9), suggesting that CsTLP8 can act as a subunit of the SCF complex and play a role in post-transcriptional regulation of target proteins. All of these findings suggest that CsTLP8 not only functions in transcriptional regulation, but also plays a role in post-transcriptional regulation. This is not unique, as the MAP kinase ERK5 possesses a kinase domain in the $\mathrm{NH}_{2}$-terminal region and a transcriptional activation domain and a nuclear localization signal in the $\mathrm{COOH}$ terminal region, allowing this protein to regulate transcription at the nucleus by either phosphorylation or interaction with transcription factors [44]. In mouse, UBE3A is known as an E3 ubiquitin ligase, which several targets have been identified, UBE3A also functions as a transcriptional regulator of the family of nuclear receptors by interaction with IRF (Interferon Regulatory Factor) [45].

In cotton, the expression of GhERF38 was up-regulated by salt and drought treatment, and over-expression of GhERF38 in Arabidopsis reduced plant tolerance to salt and drought stress[46]. The expression of $A B I 4$, a pivotal transcription factor in the ABA signaling pathway, was induced under salt stress, and ABI4 negatively regulates salt tolerance in Arabidopsis [47]. The expressions of GmLHY1a and GmLHY1b were all induced by drought stress, GmLHY1a and GmLHY1b negatively control drought tolerance in soybean [48]. All above these imply that the stress response genes, the expression was upregulated under stress conditions, might function as a negative regulator in response to stress. This is consistent with our results in this study, that the expression of CsTLP8 was induced by $\mathrm{NaCl}$ treatment, but the overexpression lines exhibited an increased susceptibility to salt stress. WRKY25, WRKY26, and WRKY33 mediate responses to heat stress by positively regulating the cooperation between the HSPs and MBF1c pathways [49], and the WRKY33-PIF4 regulatory loop also mediates $\mathrm{H}_{2} \mathrm{O}_{2}$ homeostasis, suggesting that WRKY33 responds to different stresses via different signaling pathways [50]. Similarly, CsTLP8 may function as a transcription factor or an F-box protein, which may allow CsTLP8 to participate in different signaling pathways to respond to different stresses.

\section{Conclusion}

In our study, we found that CsTLP8 functions in ABAand osmotic stress-mediated seed germination. We further confirmed that CsTLP8 regulates osmotic stressmediated seed germination by affecting antioxidant enzymes activities, and CsTLP8 participates in ABA signaling pathway. In addition, we identified that CsTLP8 could function as a transcription factor and a subunit of the SCF complex. Our work provides a new sight to study the molecular mechanism of CsTLP8 response to abiotic stress. Our work provides a new sight to study the molecular mechanism of CsTLP8 response to abiotic stress. And we will further explore the molecular mechanism of CsTLP regulating seed germination and responding to drought and salt stress.

\section{Materials and methods}

Sample preparation and total RNA extraction

Cucumber seedlings (Cucumis sativus L. Jinyan 4, from Tianjin Cucumber Research Institute, Tianjin, China. Permissions for all the materials used in this experience have been obtained) were grown in Yamasaki culture medium in a growth chamber (photoperiod: $16 \mathrm{~h}$ light $/ 8 \mathrm{~h}$ dark; temperature: $24{ }^{\circ} \mathrm{C}$ light $/ 18{ }^{\circ} \mathrm{C}$ dark; light intensity: about $125 \mu \mathrm{mol} \mathrm{m}{ }^{-2} \mathrm{~s}^{-1}$; relative humidity: about $60 \%$ ). To investigate the expression of CsTLPs under abiotic stress, 3-week-old cucumber seedlings were treated with either $150 \mathrm{mM} \mathrm{NaCl}, 10 \% \mathrm{PEG}_{6000}$, or $100 \mu \mathrm{M}$ ABA for $0 \mathrm{~h}, 1 \mathrm{~h}, 3 \mathrm{~h}, 6 \mathrm{~h}, 9 \mathrm{~h}, 12 \mathrm{~h}$, and $24 \mathrm{~h}$. The leaves from three individual plants were collected, and these samples were used to extract total RNA according to our previously published protocol [51].

\section{Quantitative real-time PCR}

Quantitative real-time PCR (qPCR) was conducted as described by Zhao et al. [52]. Basically, qPCR was performed on a 7500 Real-time PCR System (Applied Biosystems), the $2^{-\triangle \triangle \mathrm{CT}}$ quantification method was used, and CsActin was used as an inner control gene.

\section{Cloning and sequence analysis of CSTLP8}

The full-length coding sequence of CsTLP8 was amplified by PCR from cucumber CDNA using the primer pairs listed in Supplementary Table S1. Phylogenetic analyses were conducted with MEGA5. Multiple alignment analysis was performed with DNAMAN. Conserved domains were analyzed using the Pfam database (http://pfam. xfam.org/). TBtools was used to redraw the phylogenetic tree and conserved domains. The three-dimensional model of CsTLP8 was built by SWISS-MODEL (https:// swissmodel.expasy.org). The putative cis- elements present in the promoter sequence were predicted using the online program PlantCARE (http://bioinformatics.psb. ugent.be/webtools/plantcare/html/search_CARE.html).

\section{Transactivation assay of CsTLP8}

The coding sequence of CsTLP8 was inserted into the pGBKT7 vector (Addgene, Cambridge, MA, USA). The yeast strain $\mathrm{Y} 2 \mathrm{H}$ Gold [53] was transformed with pGBKT7-CsTLP8, pGBKT7-CsATAF1 (positive control) [54], or the empty vector pGBKT7 (negative control) 
as by $\mathrm{Ma}$ et al. [55]. The transformed yeast strains were spotted onto $\mathrm{SD} /-\operatorname{Trp} /$-His/X- $\alpha$-gal plates.

\section{Subcellular localization of CsTLP8}

The coding sequence of CSTLP8 (without termination codon) was inserted between CaMV $35 \mathrm{~S}$ and GFP into the pCAMBIA1302 vector (Addgene, Cambridge, MA, USA). The recombinant vector and the empty vector pCAMBIA1302 (control) were introduced into Agrobacterium tumefaciens GV3101 and then transferred to tobacco leaves. The abaxial epidermis of transgenic tobacco was analyzed by confocal microscopy (FV1000, Olympus) with bright field and fluorescence imaging. Cell nucleus was stained with DAPI (Sigma-Aldrich, D9542).

\section{Yeast two-hybrid assays}

The coding sequences of CsTLP8 and CsSKP1s were inserted into pGADT7 (Addgene, Cambridge, MA, USA) and pGBKT7 vectors, respectively, pGADT7CsTLP8 + pGBKT7-CsSKP1s, pGADT7-T + pGBKT753 (positive control) and pGADT7-CsTLP8 + pGBKT7 (negative control) were co-transformed into yeast $\mathrm{Y} 2 \mathrm{H}$ Gold cells as previously described [56]. The transformed yeast colonies were selected on DDO medium (SD/-Leu/-Trp). Single colonies of transformants growing on DDO medium were transferred to QDO medium (SD/-Trp/-Leu/-His/-Ade) supplemented with $\mathrm{X}$ - $\alpha$-gal.

\section{Yeast ectopic expression assays}

The coding sequence of CsTLP8 was inserted into the pYES2 vector (Addgene, Cambridge, MA, USA). The yeast strain W303 [57] was transformed with the recombinant vector or the empty vector pYES2. Growth assays were performed as described by Ye et al. [58], and $10 \mu \mathrm{l}$ yeast culture at different dilution ratios was dropped on YPDA (Yeast peptone dextrose adenine) medium supplemented with $250 \mathrm{mM} \mathrm{NaCl}$ or $300 \mathrm{mM}$ mannitol. After $72 \mathrm{~h}$ of culture at $30^{\circ} \mathrm{C}$, the growth situation of yeast cells was observed and recorded.

\section{Plasmid Construction and Arabidopsis transformation}

The open reading frame of CSTLP8 was inserted into the pBI121 vector (Addgene, Cambridge, MA, USA), and then the recombinant vector was integrated into Columbia wild type Arabidopsis (Col-0) by Agrobacterium tumefaciens (C58)-mediated transformation. The transgenic lines were identified by resistance to kanamycin antibiotic and PCR amplification (Fig. S2). Two transgenic lines, OE7 and OE11, were chosen for further study.

\section{Phenotype analysis}

Seed germination was conducted as previously described [59]. Briefly, 50 sterilized seeds were sown on half-strength MS medium supplemented without or with different concentrations of $\mathrm{ABA}, \mathrm{NaCl}$, or mannitol. Seeds were stratified at $4{ }^{\circ} \mathrm{C}$ in dark for 3 days and then transferred to growth chamber (photoperiod: $16 \mathrm{~h}$ light/ $8 \mathrm{~h}$ dark; temperature: $22{ }^{\circ} \mathrm{C}$ light $/ 18{ }^{\circ} \mathrm{C}$ dark; light intensity: $80 \mu \mathrm{mol} \mathrm{m} \mathrm{m}^{-2} \mathrm{~s}^{-1}$; relative humidity $60 \%$ ). After 7 days germination, the seed germination rate was calculated. In addition, seeds were sown for 4 days on half-strength MS medium and then transferred to half-strength MS medium with different concentrations of ABA, $\mathrm{NaCl}$, or mannitol for 7 days, then root length were determined.

\section{Measurement of MDA content and Antioxidant Enzymes Activities}

Arabidopsis seeds were sown for 4 days on half-strength MS medium and then transferred to half-strength MS medium with different $0.25 \mu \mathrm{M} \mathrm{ABA}, 100 \mathrm{mM} \mathrm{NaCl}$, or $100 \mathrm{mM}$ mannitol, 7 days later, seedlings were collected for measurement of MDA content and antioxidant enzymes activities. The content of malondialdehyde (MDA) as indicator of lipid peroxidation was measured according to the method of Qi et al. [60], briefly, samples were homogenized in phosphate buffer solution, then the supernatant was incubated with 5\% TBA (Thiobarbituric Acid) at $100^{\circ} \mathrm{C}$ for $10 \mathrm{~min}$. Absorbance was analyzed at 600,532 , and $450 \mathrm{~nm}$. The SOD activity was measured by monitoring the reduction in absorbance of NBT (Nitro-blue Tetrazolium) at $560 \mathrm{~nm}$, according to the method of Zhang et al. [61]. CAT activity was determined by monitoring the disappearance of $\mathrm{H}_{2} \mathrm{O}_{2}$ at $240 \mathrm{~nm}$, according to the method of Zhang et al. [61]. POD activity was determined by monitoring the oxidation of guaiacol, according to the method of Zhang et al. [61].

\section{Stomatal aperture bioassay}

Stomatal apertures were measured as previously described [54]. Briefly, fully expanded rosette leaves from 3-week-old plants were collected and incubated in buffer solution $\left(50 \mu \mathrm{M} \mathrm{CaCl}_{2}, 10 \mathrm{mM} \mathrm{KCl}, 10 \mathrm{mM}\right.$ MES (2-(N-morpholino) ethanesulfonic acid), $\mathrm{pH}$ 6.0) for $2.5 \mathrm{~h}$ in light. Then leaves were treated with ABA for $2 \mathrm{~h}$. Subsequently, the abaxial epidermal were peeled off and observed under a light microscope (Olympus-IX71). The stomatal apertures were measured using Image J, 100 stomata were measured for each sample. 


\section{Primer list}

The primers used in this study are listed in Supplementary Table S1.

\section{Statistical analysis}

Each experiment was repeated three times, and then the data were analyzed by Duncan's multiple range test $(\mathrm{P}<0.05)$ using SPSS 18.0 software (IBM Corp. Armonk, NY, USA).

\section{Abbreviations}

ABA: Abscisic Acid; ASK: Arabidopsis Skp1-like proteins; CAT: Catalase; CUL: Cullin; DAPI: 4,6-Diamidino-2-phenylindole; IRF: Interferon Regulatory Factor; MDA: Malonaldehyde; MES: 2-(N-morpholino) ethanesulfonic acid; NBT: Nitro-blue Tetrazolium; NF-YC3: Nuclear Factor Y subunit C3; PEG: Polyethylene Glycol; PIP2: Phosphatidylinositol 4, 5-bisphosphate; POD: Peroxidase; qPCR: Quantitative real-time PCR; RBX1: RING-box protein 1; ROS: Reactive Oxygen Species; SOD: Superoxide Dismutase; SKP1: S-phase kinase-associatedprotein 1; TBA: Thiobarbituric Acid; TLP/TULP: Tubby-like protein.
\end{abstract}

\section{Supplementary Information}

The online version contains supplementary material available at https://doi. org/10.1186/s12870-021-03126-y.

\section{Additional file 1: Figure S1 Three-dimensional model of CsTLP8.}

Additional file 2: Figure S2 The transgenic lines were identified by the screen of kanamycin antibiotics (a) and PCR amplification (b).

Additional file 3: Figure S3 Seeds of Col-0, OE7 and OE11 germinated on 1/2 MS media supplemented without or with $\mathrm{ABA}$, mannitol or $\mathrm{NaCl}$, Bar $=1 \mathrm{~cm}$

Additional file 4: Table S1 Primers used in this research.

Additional file 5: Table S2 List of gene accession number.

\section{Acknowledgements}

We thank the support from Engineering Research Center of Breeding and Propagation of Horticultural Crops.

\section{Authors' contributions}

SL, NZ, BZ and YG conceived this project and designed the research. SL, ZW, FW and $\mathrm{HL}$ performed most of the experiments. MC, FL, XY and HW participated in this work. BZ, $\mathrm{XL}$ and $\mathrm{SL}$ analyzed the data. $\mathrm{SL}, \mathrm{NZ}$ and $\mathrm{YG}$ wrote the article. All authors discussed the manuscript. The author(s) read and approved the final manuscript.

\section{Funding}

This work was supported by the National Key Research and Development Program of China to Bing Zhao (2019YFD1000300), the grants to Fengju Li (18ZXZYNC00170) and to Yang-Dong Guo (BAIC07 and LJNY201812). Theses funding agencies paid the experimental costs for this study but not involved in the design of the study and collection, analysis, and interpretation of data and in writing the manuscript.

\section{Availability of data and materials}

Sequence data for genes described in this study are available using the accession numbers listed in Supplementary Table S2. All data generated or analyzed during this study are included in this published article and its supplementary information files, and available from the corresponding author on reasonable request.

\section{Declarations}

Ethics approval and consent to participate

Not applicable.

\section{Consent for publication}

Not applicable.

\section{Competing interests}

The authors declare no conflict of interests.

\section{Author details}

${ }^{1}$ Beijing Key Laboratory of Growth and Developmental Regulation for Protected Vegetable Crops, College of Horticulture, China Agricultural University, Beijing 100193, China. ${ }^{2}$ Beijing Academy of Forestry and Pomology Sciences, Beijing Academy of Agriculture and Forestry Sciences, Beijing 100093, China. ${ }^{3}$ Tianjin Academy of Agricultural Sciences, 300192 Tianjin, China. ${ }^{4}$ Shandong Provincial Key Laboratory of Cucurbitaceae Vegetable Biological Breeding,

Shandong Huasheng Agriculture Co. Ltd, Qingzhou 262500, Shandong, China.

Received: 29 March 2021 Accepted: 6 July 2021

Published online: 17 July 2021

\section{References}

1. Kleyn PW, Fan W, Kovats SG, Lee JJ, Pulido JC, Wu Y, et al. Identification and characterization of the mouse obesity gene tubby: A member of a novel gene family. Cell. 1996;85:281-90.

2. NobenTrauth K, Naggert JK, North MA, Nishina PM. A candidate gene for the mouse mutation tubby. Nature. 1996;380:534-8.

3. Liu QP. Identification of rice TUBBY-like genes and their evolution. FEBS J. 2008:275:163-71.

4. Boggon TJ, Shan WS, Santagata S, Myers SC, Shapiro L. Implication of tubby proteins as transcription factors by structure-based functional analysis. Science. 1999;286:2119-25.

5. Bai C, Richman R, Elledge SJ. Human Cyclin-F. EMBO J. 1994;13:6087-98.

6. Abd-Hamid NA, Ahmad-Fauzi MI, Zainal Z, Ismail I. Diverse and dynamic roles of F-box proteins in plant biology. Planta. 2020;251:31.

7. Bao Y, Song W, Jin Y, Jiang C, Yang Y, Li B, et al. Characterization of Arabidopsis Tubby-like proteins and redundant function of AtTLP3 and AtTLP9 in plant response to ABA and osmotic stress. Plant Mol Biol. 2014;86:471-83.

8. Lai C, Shaw J. Interaction analyses of Arabidopsis tubby-like proteins with ASK proteins. Bot Stud. 2012;53:447-58.

9. Lai CP, Lee CL, Chen PH, Wu SH, Yang CC, Shaw JF. Molecular analyses of the Arabidopsis TUBBY-like protein gene family. Plant Physiol. 2004;134:1586-97.

10. Hong M, Kim D, Seo Y. Interactions between wheat Tubby-like and SKP1like proteins. Genes Genet Syst. 2015;90:293-304.

11. Wardhan V, Jahan K, Gupta S, Chennareddy S, Datta A, Chakraborty $\mathrm{S}$, et al. Overexpression of CaTLP1, a putative transcription factor in chickpea (Cicer arietinum L.), promotes stress tolerance. Plant Molecular Biology. 2012;79:479-93

12. Kou Y, Qiu D, Wang L, Li X, Wang S. Molecular analyses of the rice tubbylike protein gene family and their response to bacterial infection. Plant Cell Rep. 2009;28:113-21.

13. Cai M, Qiu D, Yuan T, Ding X, Li H, Duan L, et al. Identification of novel pathogen-responsive cis-elements and their binding proteins in the promoter of OsWRKY13, a gene regulating rice disease resistance. Plant, Cell Environ. 2008;31:86-96.

14. Albuquerque E, Petitot A, da Silva J, Grossi-de-Sa M, Fernandez D. Early responses of coffee immunity-related genes to root-knot nematode infection. Physiol Mol Plant Pathol. 2017;100:142-50.

15. Muthiah M, Ramadass A, Amalraj RS, Palaniyandi M, Rasappa V. Expression profiling of transcription factors (TFs) in sugarcane $X$ Colletotrichum falcatum interaction. J Plant Biochem Biotechnol. 2013;22:286-94.

16. Gharaghani F, Rafiei F, Mirakhorli N, Ebrahimie E. Differential expression of TLP, ERF1, and R2R3MYB in annual Medicago species under salinity conditions. Genet Mol Res. 2015;14:10152-64. 
17. Janiak A, Kwasniewski M, Sowa M, Kuczynska A, Mikolajczak K, Ogrodowicz P, et al. Insights into Barley Root Transcriptome under Mild Drought Stress with an Emphasis on Gene Expression Regulatory Mechanisms. Int J Mol Sci. 2019;20:6139.

18. Chen Y, Dai W, Sun B, Zhao Y, Ma Q. Genome-wide identification and comparative analysis of the TUBBY-like protein gene family in maize. Genes \& Genomics. 2016;38:25-36.

19. Dong $M$, Fan $X$, Pang $X$, Li Y. Decrypting tubby-like protein gene family of multiple functions in starch root crop cassava. AoB Plants. 2019;11:plz075

20. Xu J, Xing S, Zhang Z, Chen X, Wang X. Genome-Wide Identification and Expression Analysis of the Tubby-Like Protein Family in the Malus domestica Genome. Front Plant Sci. 2016;7:1693.

21. Du F, Xu J, Zhan C, Yu Z, Wang X. An obesity-like gene MdTLP7 from apple (Malus $x$ domestica) enhances abiotic stress tolerance. Biochem Biophys Res Commun. 2014:445:394-7.

22. Xu J, Xing S, Sun Q, Zhan C, Liu X, Zhang S, et al. The expression of a tubby-like protein from Malus domestica (MdTLP7) enhances abiotic stress tolerance in Arabidopsis. BMC Plant Biol. 2019;19:60.

23. Wardhan V, Pandey A, Chakraborty S, Chakraborty N. Chickpea transcription factor CaTLP1 interacts with protein kinases, modulates ROS accumulation and promotes ABA-mediated stomatal closure. Sci Rep. 2016;6:38121.

24. Reitz M, Bissue J, Zocher K, Attard A, Huckelhoven R, Becker K, et al. The Subcellular Localization of Tubby-Like Proteins and Participation in Stress Signaling and Root Colonization by the Mutualist Piriformospora indica. Plant Physiol. 2012;160:349-64.

25. Wang M, Xu Z, Ahmed R, Wang Y, Hu R, Zhou G, et, al. Tubby-like Protein 2 regulates homogalacturonan biosynthesis in Arabidopsis seed coat mucilage. Plant Molecular Biology. 2019;99:421-36

26. Mohnen D. Pectin structure and biosynthesis. Curr Opin Plant Biol. 2008:11:266-77.

27. Song W, Cheng Z, Guo X, Yu C, Wang H, Wang J, et al. Overexpression of NHL6 affects seed production in transgenic Arabidopsis plants. Plant Growth Regulation. 2019;88:41-7.

28. Bao Y, Song W, Pan J, Jiang C, Srivastava R, Li B, et al. Overexpression of the NDR1/HIN1-Like Gene NHL6 Modifies Seed Germination in Response to Abscisic Acid and Abiotic Stresses in Arabidopsis. Plos One. 2016;11:e0148572.

29. Myskow B, Goralska M, Lenarczyk N, Czyczylo-Mysza I, Stojalowski S. Putative candidate genes responsible for leaf rolling in rye (Secale cereale L.). BMC Genetics. 2018;19:57

30. Zhang Y, He X, Su D, Feng Y, Zhao H, Deng H, et al. Comprehensive Profiling of Tubby-Like Protein Expression Uncovers Ripening-Related TLP Genes in Tomato (Solanum lycopersicum). Int J Mol Sci. 2020;21:1000.

31. Li S, Zhang J, Liu L, Wang Z, Li Y, Guo L, et al. SITLFP8 reduces water loss to improve water-use efficiency by modulating cell size and stomatal density via endoreduplication. Plant Cell Environ. 2020;43(11):2666-79.

32. Guo G, Liu X, Sun F, Cao J, Huo N, Wuda B, et al. Wheat miR9678 affects seed germination by generating phased sirnas and modulating abscisic acid/gibberellin signaling. Plant Cell. 2018;30(4).

33. Hu Y, Han $X$, Yang $M$, Zhang M, Pan J, Yu D. The transcription factor INDUCER OF CBF EXPRESSION1 interacts with ABSCISIC ACID INSENSITIVE5 and DELLA proteins to fine-tune abscisic acid signaling during seed germination in Arabidopsis. Plant Cell. 2019;31(7).

34. Tan L, Chen S, Wang T, Dai S. Proteomic insights into seed germination in response to environmental factors. Proteomics. 2013;13(12-13).

35. Lotfi N, Soleimani A, Vahdati K, Çakmakçi R. Comprehensive biochemical insights into the seed germination of walnut under drought stress. Sci Hortic. 2019;250:329-43.

36. Bai Y, Xiao S, Zhang Z, Zhang Y, Sun H, Zhang K, et al. Melatonin improves the germination rate of cotton seeds under drought stress by opening pores in the seed coat. Peer J. 2020;8.

37. Mwando E, Angessa TT, Han Y, Li C. Salinity tolerance in barley during germination- homologs and potential genes. J Zhejiang Univ Sci B. 2020;21(2):93-121.

38. Karimi S, Karami H, Mokhtassi-Bidgoli A, Tavallali V, Vahdati K. Inducing drought tolerance in greenhouse grown Juglans regia by imposing controlled salt stress: The role of osmotic adjustment. Scientia Horticulturae, 2018,239.

39. Santagata S, Boggon TJ, Baird CL, Gomez CA, Zhao J, Shan WS. et, al. G-protein signaling through tubby proteins Science. 2001;292:2041-50.
40. Wang $M$, Jiang $B$, Peng QW, Liu WR, He XM, Liang ZJ, et al. Transcriptome analyses in different cucumber cultivars provide novel insights into drought stress responses. Int J Mol Sci. 2018;19:15.

41. Ma Z, Natalia VB, Abir UI. Cell signaling mechanisms and metabolic regulation of germination and dormancy in barley seeds. The Crop Journal. 2017;5(06):459-77.

42. Moller IM, Jensen PE, Hansson A. Oxidative modifications to cellular components in plants. Annu Rev Plant Biol. 2007;58:459-81.

43. Vahdati K. Walnut tolerance to abiotic stresses: approaches and prospects. Acta Hort. 2014;1050:399-406.

44. Erazo T, Espinosa-Gil S, Dieguez-Martinez N, Gomez N, Lizcano JM. SUMOylation is required for ERK5 nuclear translocation and ERK5-mediated cancer cell proliferation. Int J Mol Sci. 2020;21:2203.

45. Furumai $R$, Tamada K, Liu XX, Takumi T. UBE3A regulates the transcription of IRF, an antiviral immunity. Hum Mol Genet. 2019;28:1947-58.

46. Ma L, Hu L, Fan J, Amombo E, Khaldun B, Zheng Y, et al. Cotton GhERF38 gene is involved in plant response to salt/drought and ABA. Ecotoxicol. 2017;26(6).

47. Kakan X, Yu Y, Li S, Li X, Huang R, Wang J. Ascorbic acid modulation by ABI4 transcriptional repression of VTC2 in the salt tolerance of Arabidopsis. BMC Plant Biol. 2021;21(1).

48. Wang K, Bu T, Cheng Q, Dong L, Su T, Chen Z, et al. Two homologous LHY pairs negatively control soybean drought tolerance by repressing the abscisic acid responses. New Phytologist, 2020,229(5).

49. Li S, Fu Q, Chen L, Huang W, Yu D. Arabidopsis thaliana WRKY25, WRKY26, and WRKY33 coordinate induction of plant thermotolerance. Planta. 2011;233:1237-52

50. Sun YJ, Liu ZX, Guo JG, Zhu ZN, Zhou YP, Guo CX, et al. WRKY33-PIF4 loop is required for the regulation of $\mathrm{H}_{2} \mathrm{O}_{2}$ homeostasis. Biochem Biophys Res Commun. 2020;527:922-8.

51. Cao YY, Qi CD, Li ST, Wang ZR, Wang XY, Wang JF, et al. Melatonin Alleviates Copper Toxicity via Improving Copper Sequestration and ROS Scavenging in Cucumber. Plant Cell Physiol. 2019;60:562-74.

52. Zhao L, Li MM, Xu CJ, Yang X, Li DM, Zhao X, et al. Natural variation in GmGBP1 promoter affects photoperiod control of flowering time and maturity in soybean. Plant J. 2018;96:147-62.

53. Wang Y, Li Y, He SP, Gao Y, Wang NN, Lu R, et al. A cotton (Gossypium hirsutum) WRKY transcription factor (GhWRKY22) participates in regulating anther/pollen development. Plant Physiol Biochem. 2019;141:231-9.

54. Wang J, Zhang L, Cao Y, Qi C, Li S, Liu L, et al. CSATAF1 positively regulates drought stress tolerance by an ABA-dependent pathway and by promoting ROS scavenging in cucumber. Plant Cell Physiol. 2018;59:930-45.

55. Ma HZ, Liu C, Li ZX, Ran QJ, Xie GN, Wang BM, et al. ZmbZIP4 Contributes to Stress Resistance in Maize by Regulating ABA Synthesis and Root Development. Plant Physiol. 2018;178:753-70.

56. Li CH, Chiang CP, Yang JY, Ma CJ, Chen YC, Yen HE. RING-type ubiquitin ligase MCCPN1 catalyzes UBC8-dependent protein ubiquitination and interacts with Argonaute 4 in halophyte ice plant. Plant Physiol Biochem. 2014;80:211-9.

57. Fiorentini P, Huang KN, Tishkoff DX, Kolodner RD, Symington LS. Exonuclease I of Saccharomyces cerevisiae functions in mitotic recombination in vivo and in vitro. Mol Cell Biol. 1997;17:2764-73.

58. Ye YY, Lin RY, Su HX, Chen HF, Luo M, Yang LX, et al. The functional identification of glycine-rich TtASR from Tetragonia tetragonoides (Pall.) Kuntze involving in plant abiotic stress tolerance. Plant Physiol Biochem. 2019;143:212-23.

59. Zhao R, Sun HL, Mei C, Wang XJ, Yan L, Liu R, et, al. The Arabidopsis $\mathrm{Ca}^{2+}$-dependent protein kinase CPK12 negatively regulates abscisic acid signaling in seed germination and post-germination growth. New Phytologist. 2011;192:61-73

60. Qi C, Zhang H, Liu Y, Wang X, Dong D, Zhang N, et al. CsSNAT positively regulates salt tolerance and growth of cucumber by promoting melatonin biosynthesis. Environ Exper Botany. 2020;175.

61. Zhang W, Xia X, Wang J, Zhu L, Wang J, Wang G, et al. Oxidative stress and genotoxicity of nitenpyram to earthworms (Eisenia foetida). Chemosphere. 2021;264(P2).

\section{Publisher's Note}

Springer Nature remains neutral with regard to jurisdictional claims in published maps and institutional affiliations. 